\title{
Impacto de las asincronías en el pronóstico del paciente ventilado
} Impact of asynchronies on the prognosis of the ventilated patient Impacto das assincronias no prognóstico do paciente ventilado

Alexéi Humberto Morales Morales, ${ }^{\star}$ José Carlos Gasca Aldama, ${ }^{*}$ Karen Josefina Castillo Medrano, ${ }^{*}$ Sandybell Sosa Santos ${ }^{*}$

\section{RESUMEN}

La alta incidencia de asincronías paciente-ventilador puede modificar el pronóstico del paciente. A pesar de tener una incidencia reportada de 10 al $85 \%$, las asincronías no se diagnostican con frecuencia. Éstas ocurren cuando existe un desajuste con respecto a las demandas de tiempo, flujo, volumen o presión del sistema respiratorio del paciente y el ventilador; se clasifican de acuerdo con la fase del ciclo respiratorio en donde ocurra. Los tipos más frecuentes son: esfuerzo inefectivo (EI) y doble disparo (DD). Están asociadas con peores resultados en general, mayor disconfort y disnea, que llevan al aumento del gasto energético y patrón diafragmático anormal asociado con lesión de los músculos respiratorios, así como con mayor riesgo de delirio, el cual culmina en la dificultad para el destete, mayor uso de sedación y bloqueadores neuromusculares, ventilación mecánica prolongada, mayor estancia en la Unidad de Cuidados Intensivos (UCI), mayor necesidad de traqueostomía y mortalidad. Actualmente se desconoce si la detección y tratamiento oportuno de las asincronías pueden ayudar a mejorar e pronóstico de los pacientes o representan un marcador de gravedad derivado de la alteración clínica respiratoria y de las condiciones mecánicas.

Palabras clave: Asincronías, ventilación mecánica, pronóstico.

\section{ABSTRACT}

High incidence of patient-ventilator asynchrony could modify patient prognostic. Although there is a 10 to $85 \%$ rate incidence they are not frequently diagnosed. Asynchronies occur when there is a mismatch between time, flow, volume and pressure patient respiratory demands and ventilator setting, they are classified according to respiratory cycle time, most common types are ineffective effort (IE) and double triggering (DD). Asynchrony is associated to worst outcomes, related to discomfort and dyspnea that leads to increasing wasting expenditure energy and abnormal diaphragmatic pattern associated to respiratory muscles damage as well as increasing risk of delirium which results in difficult weaning, deep sedation and use of neuromuscular blockers, longer duration of mechanical ventilation, longer stay in $\mathrm{UCl}$, increasing need of tracheotomy and higher mortality. There is not enough evidence that supports if detection and treatment of asynchronies may improve outcomes or if they belong to a severity marker that represents respiratory and mechanical alterations.

Keywords: Asynchrony, mechanical ventilation, prognostic.

\section{RESUMO}

A alta incidência de assincronias paciente-ventilador pode modificar o prognóstico do paciente. Apesar de ter uma incidência relatada de 10 a 85\%, eles não são diagnosticados com frequência. As assincronias ocorrem quando há uma incompatibilidade em relação às demandas de tempo, fluxo, volume ou pressão do sistema respiratório do paciente e do ventilador, são classificadas de acordo com a fase do ciclo respiratório em que ocorre e os tipos mais frequentes são esforço ineficaz (EI) e duplo disparo (DD). A assincronia está associada a piores resultados em geral, maior desconforto e dispnéia que levam ao aumento do gasto energético e padrão diafragmático anormal associado à lesão muscular respiratória, além de aumento do risco de delirium que culmina com dificuldade no desmame, aumento do uso de sedação e bloqueadores ventilação mecânica neuromuscular prolongada, permanência prolongada na unidade de terapia intensiva (UTI), maior necessidade de traqueostomia e mortalidade. Atualmente, não se sabe se a detecção e o tratamento oportuno das assincronias podem ajudar a melhorar o prognóstico dos pacientes ou representar um marcador de gravidade derivado de distúrbios clínicos respiratórios e condições mecânicas.

Palavras-chave: Assincronias, ventilação mecânica, previsão.

\footnotetext{
* Unidad de Cuidados Intensivos. Hospital Juárez de México. Ciudad de México.
}

Abreviaturas:

$\mathrm{EI}=$ Esfuerzo inefectivo; $\mathrm{DD}$ = Doble disparo; $\mathrm{ACV}=$ Assist-control ventilation $\mathrm{PSV}=$ Ventilación con presión de soporte; $\mathrm{VCV}=$ Ventilación controlada por volumen; VCP $=$ Ventilación Controlada por Presión

Recepción: 02/09/2019. Aceptación: 29/09/2019.

Este artículo puede ser consultado en versión completa en www.medigraphic.com/medicinacritica

\section{INTRODUCCIÓN}

La ventilación mecánica es una medida común de soporte aplicada al paciente crítico. ${ }^{1}$ Uno de sus objetivos es reducir el trabajo respiratorio del paciente al entregar un nivel suficiente de soporte ventilatorio, además de evitar la disfunción diafragmática inducida por el ventilador al permitir que el paciente genere esfuerzos espontáneos. ${ }^{2}$ Una óptima interacción entre el paciente y el ventilador es crucial para asegurar el confort durante la misma interacción y evitar efectos adversos. ${ }^{1}$

Se define a la sincronía entre el paciente y el ventilador como la interacción apropiada entre los dos, donde el ventilador reconoce el esfuerzo del paciente y brinda apoyo para respirar en el momento adecuado, es decir, el tiempo inspiratorio y de espiración coinciden con los tiempos del paciente (el tiempo neural del paciente es igual al tiempo del ventilador). ${ }^{3}$

Las asincronías ocurren cuando la asistencia del ventilador no coincide con la demanda del paciente. La asistencia ventilatoria, ya sea excesiva o insuficiente, se traduce en diferentes tipos de asincronías con diferentes efectos en los pacientes; ${ }^{1}$ éstas son eventos frecuentes y variables, presentes durante todo el periodo de ventilación mecánica y están asociadas con peores resultados clínicos, como disconfort y disnea; además se incrementa la necesidad de agentes bloqueadores y sedantes, ventilación mecánica prolongada, más días de estancia en la Unidad de Cuidados Intensivos y en el hospital, lesión de los músculos respiratorios, mayor necesidad de traqueostomía y mortalidad (Figura 1).4-8

Dentro de los factores relacionados con el paciente se encuentran: la sepsis, acidosis, ansiedad y fiebre, los cuales incrementan la demanda ventilatoria e impiden el equilibrio entre la demanda de flujo y volumen exigido por el paciente y lo entregado por el ventilador. ${ }^{9}$ La enfermedad pulmonar obstructiva crónica ha sido considerada la condición más comúnmente asociada, especialmente en presencia de auto-PEEP (auto-positive end expiratory pressure, por sus siglas en inglés) lo que dificulta la activación del ventilador y favorece los esfuerzos inefectivos. ${ }^{9}$ Otros factores relacionados son: la indicación de ventilación mecánica, la severidad de la falla respiratoria, el modo ventilatorio, los ajustes del ventilador y el nivel de sedación. En cambio, los factores relacionados con la detección de las asincronías son: el tiempo y los periodos de observación, el método de detección (mediciones clínicas, monitorización de las curvas de ventilación, medición mediante balón 
esofágico o detección de la actividad eléctrica del diafragma) y la definición per se de asincronías y su significado. ${ }^{9}$

En su estudio, Arnaud W. Thille y colaboradores, en 2006, encontraron que una cuarta parte de los pacientes presentó una alta incidencia de asincronías durante la asistencia ventilatoria y que se asoció con una mayor duración de la ventilación mecánica. ${ }^{2}$ Recientemente, Blanch y su equipo usaron un software para estudiar la prevalencia y el curso en el tiempo de cinco tipos de asincronías: esfuerzo inspiratorio inefectivo, doble disparo, inspiraciones abortadas y ciclado prematuro y tardío. La mediana en el índice de asincronía por paciente durante todo el curso de ventilación mecánica fue de $3.41 \%$. Se produjeron asincronías durante todo el periodo de ventilación mecánica, todo el día, incluso en modo controlado, y se asociaron con mortalidad. Como en estudios previos, el esfuerzo inspiratorio inefectivo fue la asincronía más común en todos los modos. ${ }^{4,5}$

La asociación entre asincronía y pronóstico existe; los pacientes con un índice de asincronía (IA) > 10\% (definido por el número de eventos dividido entre el número total de ciclos ventilatorios y multiplicado por 100) están bajo ventilación mecánica un periodo de tiempo más largo y presentan mayor mortalidad, tanto en la UCl como en el hospital. Sin embargo, todavía no se puede estar seguros de si el control de las asincronías puede ayudar a mejorar el pronóstico de los pacientes o si son simplemente un marcador de gravedad derivado de la alteración clínica respiratoria y de las condiciones mecánicas. ${ }^{3}$

Una nueva investigación también ha explorado los efectos psicológicos de las asincronías. La ansiedad y la depresión son comunes en los sobrevivientes de enfermedades críticas incluso después del alta. Los pacientes con ventilación mecánica sienten ansiedad,

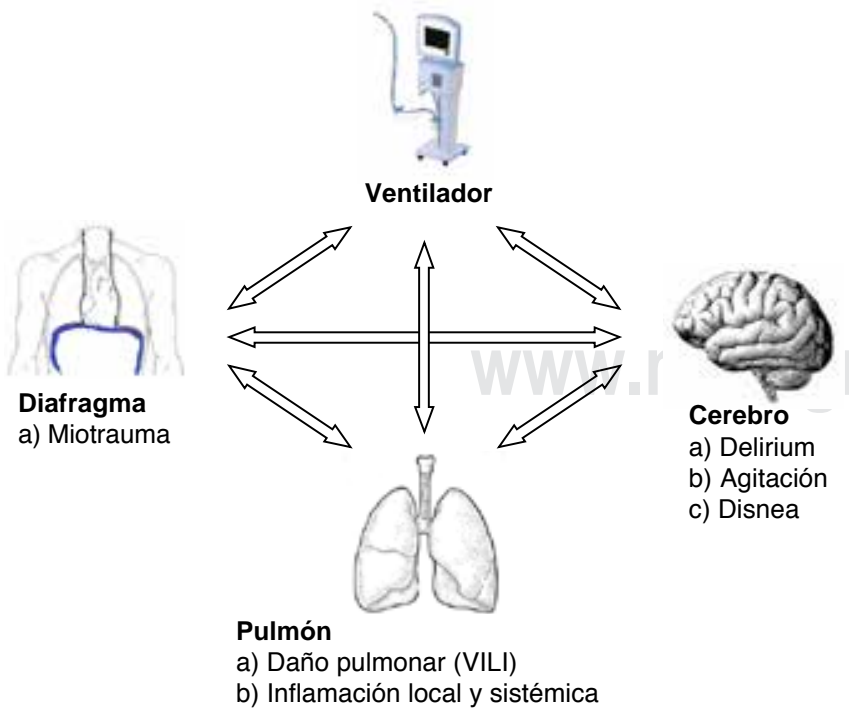

Figura 1: Interacciones de las asincronías y sus consecuencias. Elaboración propia.

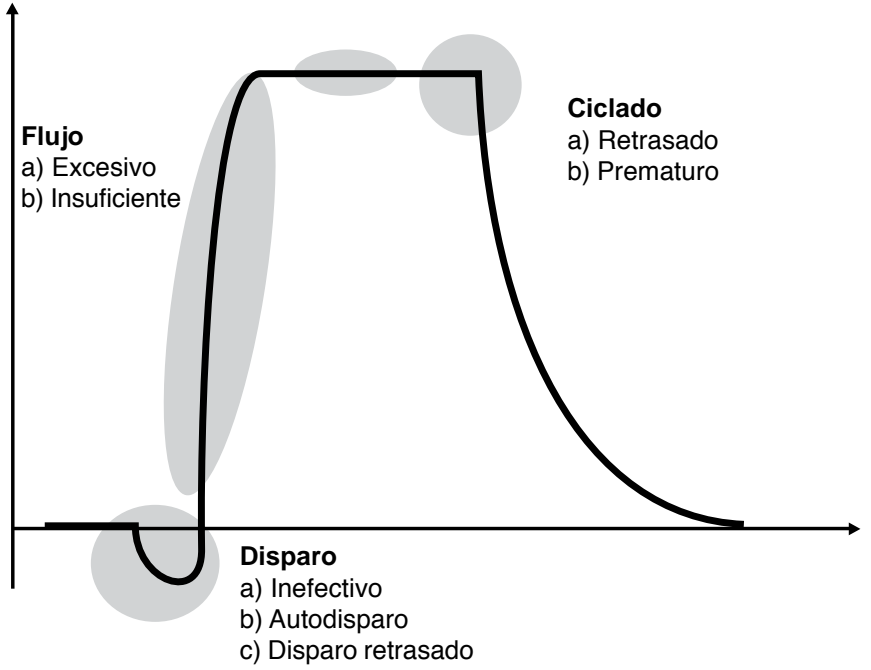

Figura 2: Las asincronías se pueden clasificar según la fase del ciclo donde se producen: durante la fase inspiratoria, durante la transición de inspiración a espiración o durante la espiración.

miedo, agonía e inseguridad, que pueden empeorar con la presencia de asincronías. ${ }^{1}$

Por lo tanto, diagnosticar y corregir las asincronías debe ser una prioridad en todo momento de la ventilación mecánica. ${ }^{4}$

\section{Manifestaciones clínicas y hemodinámicas}

Los signos clínicos de dificultad respiratoria/asincronías son los siguientes: 6,10

Taquipnea, uso de músculos accesorios, diaforesis, movimiento diafragmático paradójico, retracción subdiafragmática, retracción supraesternal, supraclavicular o intercostal, cianosis, taquicardia y disminución en la saturación de $\mathrm{O}_{2}$.

Sabemos que el corazón y los pulmones están vinculados anatómica y funcionalmente. Los efectos hemodinámicos de la ventilación y las asincronías dependen del estado previo del sistema cardiopulmonar. En pacientes con ventilación mecánica, el aumento inspiratorio de la presión intratorácica reduce el retorno venoso al aumentar la presión auricular derecha, y reduce la postcarga ventricular izquierda al disminuir la presión sistólica transmural del ventrículo izquierdo. Por el contrario, puede aumentar considerablemente la postcarga del ventrículo derecho, que resulta de aumentos progresivos en la presión transpulmonar asociados con el aumento del volumen pulmonar. ${ }^{1}$

Los efectos hemodinámicos causados por los diferentes tipos de asincronías no se han estudiado ampliamente. En teoría, los esfuerzos ineficaces disminuyen la presión intratorácica y, en consecuencia, pueden au- 


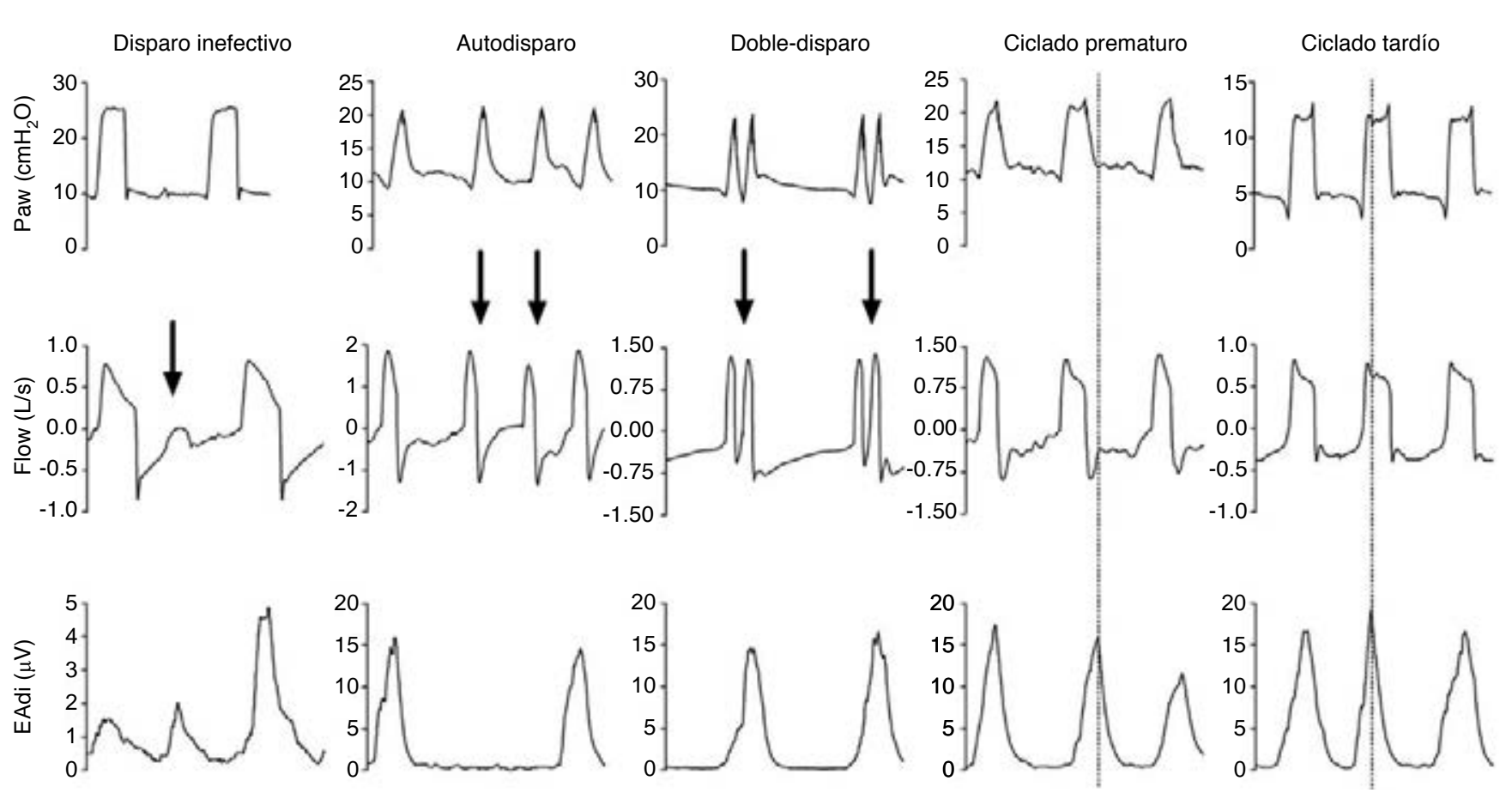

Figura 3: Tipos de asincronías. Modificado de: Garofalo E, Bruni A, Pelaia C, Liparota L, Lombardo N, Longhini F, et al. Recognizing, quantifying and managing patient-ventilator asynchrony in invasive and noninvasive ventilation. Expert Rev Respir Med. 2018;12(7):557-567.

mentar el retorno venoso y el llenado ventricular derecho. El volumen corriente acumulado durante el doble ciclado es muy alto y la presión máxima de la segunda respiración es generalmente mayor que en la primera. Tanto el aumento del volumen como la presión podrían afectar significativamente la precarga y la postcarga, pero no se han evaluado las consecuencias hemodinámicas de estos efectos. ${ }^{1,11}$

En el estudio de Messina y su equipo, ${ }^{11}$ se evaluó la respuesta a líquidos en 54 pacientes manejados con ventilación en presión soporte (VPS) mediante la variación de presión de pulso (VPP), y se encontró que la predicción de la VPP estaba influenciada por la asincronía paciente-ventilador (OR, 8.8 [2.0-38.0]; $p<$ 0.003).

\section{Tipos de asincronías}

Los tipos de asincronías pueden dividirse en varias formas, una de ellas ocurre de acuerdo con el ciclo respiratorio en donde sucede (Figura 2): ${ }^{10}$

Periodo inspiratorio: trigger retrasado, desajuste del flujo inspiratorio, ciclado prematuro, ciclado tardío y trigger reverso.

Durante la transición de inspiración a espiración: doble trigger debido a ciclado corto o trigger reversoy contracción muscular espiratoria debido a ciclado prolongado
Periodo espiratorio: esfuerzo inspiratorio inefectivo, auto-trigger y contracción muscular espiratoria

Otra clasificación, algo más arbitraria las divide en dos grandes grupos (Figura 3):7,12

Mayores: disparo inefectivo, autodisparo y doble disparo Menores: ciclado prematuro y ciclado tardío

\section{Manejo de las asincronías}

Las estrategias de manejo de las asincronías están determinadas por el tipo y etiología de las mismas. En el siguiente cuadro se muestran las acciones específicas a realizar: ${ }^{10}$

\begin{tabular}{ll} 
Asincronía & Acción \\
\hline Desajuste del & Aumentar el flujo, disminuir el impulso respiratorio y evaluar \\
flujo inspiratorio & una adecuada analgesia y sedación; comprobar disnea \\
$\begin{array}{l}\text { Ciclado corto o } \\
\text { prolongado }\end{array}$ & $\begin{array}{l}\text { Aumentar o disminuir el tiempo inspiratorio; comprobar } \\
\text { el ciclo en presión soporte; usar modos proporcionales y } \\
\text { eliminar fugas }\end{array}$
\end{tabular}

Doble trigger

Aumentar el tiempo inspiratorio del ventilador; probar en presión soporte, valorar los criterios de terminación del flujo para mejorar sincronía o modos proporcionales; considerar agentes paralizantes si el volumen corriente es demasiado elevado (> $8 \mathrm{~mL} / \mathrm{kg} /$ peso predicho) en SIRA o en pacientes con factores de riesgo para desarrollar lesión pulmonar

Doble trigger debido a trigger reverso y considerar agentes paralizantes si el volumen corriente es demasiado elevado ( $>8 \mathrm{~mL} / \mathrm{kg} /$ peso predicho) en SIRA o en pacientes con factores de riesgo para desarrollar lesión pulmonar 


\begin{tabular}{|c|c|}
\hline Asincronía & Acción \\
\hline $\begin{array}{l}\text { Contracción mus- } \\
\text { cular espiratoria } \\
\text { debido a ciclado } \\
\text { prolongado }\end{array}$ & $\begin{array}{l}\text { Reducir el periodo inspiratorio verificando el ciclo y el volu- } \\
\text { men corriente. Verificar si hay disnea }\end{array}$ \\
\hline $\begin{array}{l}\text { Esfuerzo inefecti- } \\
\text { vo inspiratorio }\end{array}$ & $\begin{array}{l}\text { Optimizar el disparador inspiratorio y el atrapamiento de aire } \\
\text { excesivo; comprobar si hay asistencia excesiva (frecuencia } \\
\text { respiratoria y/o tiempo inspiratorio excesivos durante modos } \\
\text { controlados o de presión soporte); corregir auto-PEEP } \\
\text { mediante el uso de PEEP externo; verificar si hay disnea; } \\
\text { considerar modos proporcionales }\end{array}$ \\
\hline Auto-trigger & $\begin{array}{l}\text { Ajustar el disparador al mínimo } \\
\text { Eliminar fugas y/o agua en el circuito del ventilador }\end{array}$ \\
\hline $\begin{array}{l}\text { Contracción } \\
\text { muscular espira- } \\
\text { toria durante la } \\
\text { espiración }\end{array}$ & $\begin{array}{l}\text { Verificar la asistencia excesiva } \\
\text { Comprobar si hay atrapamiento de aire y auto-PEEP }\end{array}$ \\
\hline
\end{tabular}

\section{Impacto de las asincronías en el pronóstico}

El interés en las asincronías ha incrementado en los últimos 10 años; algunos estudios han reportado que son más comunes de lo que se espera y se han asociado con un mal pronóstico. ${ }^{10}$

A pesar de existir numerosas publicaciones que nos ayudan a identificar, clasificar y tratar las asincronías, aún no se ha podido describir el impacto que éstas tienen en el pronóstico del paciente ventilado. Como ya se explicó previamente, existen múltiples factores por los que las asincronías causan incremento de la estancia en la $\mathrm{UCl}$, días de ventilación y aumento de la mortalidad. Sin embargo, no se ha demostrado una relación causal directa entre la interacción paciente-ventilador y la evidencia que demuestre que reducir las asincronías garantice un mejor pronóstico. ${ }^{13}$

Uno de los primeros estudios que describió la incidencia y consecuencias de las asincronías en pacientes ventilados fue escrito por W. Thille y otros en el $2006 .^{2}$ En éste observaron 62 pacientes en un periodo de 30 minutos, y las principales asincronías detectadas fueron el esfuerzo inefectivo y doble disparo. Además, hubo una media de 72 pacientes en modo ACV y 16 pacientes en modo PSV, asociándose con un incremento de los días de ventilación mecánica ( 7 vs 25 días, $p=0.005)$, así como un incremento de la mortalidad (32\% vs $47 \%, p=0.36$ ). Una de las limitaciones de este estudio fue la metodología para la detección de asincronías, al efectuarse este diagnóstico por experiencia del médico tratante, lo que limita la identificación de las mismas por tiempo o por reconocimiento; además, sólo se estudiaron dos tipos de asincronías. ${ }^{1,13}$

En el 2009, De Wit y colaboradores ${ }^{2}$ realizaron un estudio prospectivo con 60 pacientes, analizando la mecánica ventilatoria por 10 minutos en las primeras 24 horas de estancia en la $\mathrm{UCl}$ y obteniendo un índice de trigger inefectivo (ITI) (número de respiraciones con disparo inefectivo dividido entre el número total de respiraciones). Así, corroboraron las asociaciones previas, un ITI $>10 \%$ fue un factor independiente de aumento de días de ventilación mecánica (10 días vs 4 días con una $p=0.0004$ ).

En el 2015, Blanch y su equipo ${ }^{4,13}$ realizaron un estudio prospectivo y observacional de 50 pacientes, en el que, con ayuda de un software instalado en el ventilador mecánico, lograron describir con precisión el índice de asincronías durante una hora continua. Fueron analizados 7,027 horas en 16 equipos. Se investigó la presencia de esfuerzo inspiratorio inefectivo (EI) y doble disparo en VCV, PCV y PSV, así como ciclado corto, prolongado y autodisparo en PSV. Los resultados obtenidos en este estudio siguen siendo al día de hoy el marco para la pronta identificación y tratamiento de las asincronías, ya que se demostró que un índice de asincronías $>10 \%$ tiene un alto impacto en la mortalidad ( $23 \%$ vs $67 \%, p=0.044$ ), días de ventilación mecánica ( 6 días vs 16 días, $p=0.061)$, reintubación ( $20 \%$ vs $0 \%$, $p=0.57$ ), traqueostomía (32\% vs 33\%, $p=0.999$ ).

El índice de asincronías, incorporado al software del ventilador mecánico mejoró la precisión de la detección de asincronías. Recientemente se han descrito nuevos métodos para la detección de eventos asincrónicos paciente-ventilador. En el 2016, Vaporidi y su equipo ${ }^{13,14}$ analizaron los datos de 110 pacientes en su primer día de ventilación asistida, sin embargo, a diferencia de los estudios previos, no se consideró un IA, y en su lugar se midieron en grupos de esfuerzos inefectivos, definidos como periodos de tres minutos con más de 30 eventos de El. En el análisis se identificaron 2,931 horas; en $38 \%$ de los pacientes al contrario que en estudios previos el índice de EI no se asoció con la mortalidad, sin embargo, la presencia de eventos de El durante las primeras 24 horas y su persistencia fue asociada con más días de ventilación mecánica (OR 6.4, $\mathrm{IC}_{95 \%}$ 1.1-38.3) y mortalidad (OR 20, IC ${ }_{95 \%}$ 2.3-175).

De esta manera la presencia de grupos de asincronías, es decir, eventos asincrónicos continuos en periodos cortos de tiempo, puede tener una mayor correlación con el pronóstico del paciente que los eventos esporádicos, como previamente se habían estudiado. En un estudio de De Haro y colaboradores, ${ }^{15}$ donde se analizaron la incidencia, los mecanismos e implicaciones fisiológicas de un doble disparo en 67 pacientes con monitoreo continuo por ventilador mecánico y 9,251 horas de ventilación mecánica correspondientes a 9,694,573 respiraciones, la asincronía de doble disparo ocurrió en sólo $0.6 \%$ de las respiraciones, pero todos los pacientes la tuvieron en algún momento. Ésta fue más frecuente en VCP con $0.54 \%(0.34-0.87 \%)$ que en VCV con $0.27 \%$ ( $0.19 \%$ vs $0.38 \%)$, concluyendo que, a pesar de ser infrecuente, ocurre en todos los pacientes, y que la repercusión fisiológica del doble disparo, al exceder el volumen óptimo para protección ventilatoria, llevaba al daño tisular y fatiga de músculos respirato- 
rios. Las respiraciones fueron activadas por el paciente en $65.4 \%$ y por trigger reverso (TR) (contracción diafragmática estimulada por una respiración respiratoria pasiva previa) en $34.6 \%$ de los casos.

Los esfuerzos pueden generar mayor presión de meseta y grandes volúmenes corrientes en VCV y PCV, aunque el doble disparo se reduce con sedación profun$\mathrm{da}$, los volúmenes corrientes altos potencialmente nocivos aún pueden entregarse. Esto sucede generalmente en pacientes sedados, principalmente con síndrome de insuficiencia respiratoria aguda y a menudo pasan desapercibidos. ${ }^{15} \mathrm{La}$ implicación clínica del trigger reverso depende del grado de desfase entre el ciclo mecánico y el esfuerzo muscular; si éste es prolongado puede producir un doble disparo con aumento del volumen corriente y de la presión de distensión alveolar. Ha sido descrito como una de las causas de doble disparo. En modos controlados por presión, el esfuerzo muscular será compensado con un aumento del volumen en cada ciclo del respirador. El trabajo respiratorio aumenta, pero es un esfuerzo perdido, sin rendimiento y ocasionalmente excesivo, como sucede durante el doble disparo. En el manejo de esta asincronía se ha sugerido el uso de presión de soporte y modos controlados por presión con frecuencias respiratorias bajas. La incidencia y significado del trigger reverso en el paciente crítico con ventilación mecánica está por estudiarse, si se debe a un inadecuado manejo del paciente, de la sedación, parámetros del ventilador o si es una respuesta fisiológica de adaptación a la ventilación mecánica. ${ }^{16}$

Hay estudios que han reportado que los esfuerzos inefectivos están asociados con una mayor duración de la ventilación mecánica y que inducen alteraciones del sueño. En el estudio de 1997, de Chao y colaboradores, los El se asocian con menor tasa de éxito de destete (16 vs $57 \%$ ). ${ }^{13}$ En el estudio de Camiller Rolland-Debord y su equipo, en el que se comparó NAVA vs PSV durante la fase temprana de destete, las asincronías no fueron asociadas con resultados clínicos adversos. ${ }^{17}$

Las asincronías pueden presentarse tanto en ventilación invasiva como en no invasiva (VNI). En un estudio observacional por Vignaux y colaboradores, 26 de 60 pacientes (43\%) con VNI tuvieron un IA > 10\% mediante análisis de electromiografía diafragmática. ${ }^{12}$ La pobre sincronización paciente-ventilador a menudo se observa durante la ventilación de soporte de presión (PSV) y se ha asociado con una duración prolongada de la ventilación mecánica y un mal resultado. Es difícil el reconocimiento de las asincronías en VNI únicamente con inspección visual de las curvas del ventilador. ${ }^{18}$ La actividad eléctrica diafragmática (Eadi) durante PSV mejoró la sincronización, pero no impactó en el trabajo respiratorio. ${ }^{19}$

Aunque actualmente no existe un metaanálisis que evalúen las asincronías y la mortalidad, en febrero del
2019 se publicó en la revista Minerva de Anestesiología ${ }^{7}$ un análisis de 62 estudios publicados describiendo las definiciones, variables y conclusiones de cada uno; se encontraron 10 estudios que evaluaron la relación entre la asincronías paciente-ventilador y mortalidad, de los cuales seis no encontraron asociación entre el IA $>10 \%$ y mortalidad, a diferencia de cuatro estudios, entre ellos el de Blanch, que corroboran esta asociación. ${ }^{13}$ Uno de las principales problemáticas para poder realizar una adecuada asociación entre los eventos de asincronías y la mortalidad son las diferentes formas de cuantificación del IA, las pequeñas muestras de pacientes y la falta de uniformidad para su identificación.

\section{CONCLUSIONES}

Con la información publicada hasta el día de hoy aún no podemos asegurar que el control de las asincronías pueda ayudarnos a mejorar el pronóstico de los pacientes o si son únicamente marcadores de severidad derivados de condiciones mecánicas, ventilatorias y/o fisiopatológicas. Sin embargo, es indudable la repercusión que tienen en los pacientes debido al incremento del trabajo respiratorio, disnea y aumento de sedación, que lleva a la debilidad diafragmática, delirio y que culmina en estancia prolongada en la $\mathrm{UCl}$, más días de ventilación mecánica y eventualmente un mayor riesgo de un desenlace fatal.

Si la lógica de la fisiopatología impera podríamos afirmar que, si existe una relación causal entre asincronías y mortalidad, faltan estudios que demuestren estadísticamente esta afirmación.

Parece intuitivamente importante mejorar la detección de asincronías y adaptar la asistencia del ventilador. ${ }^{17}$ Ewan C. Goligher menciona acertadamente que el arte de la ventilación mecánica es la sincronización entre el paciente y el ventilador, y en efecto se ha vuelto muy complejo. ${ }^{20}$ Lo que es incuestionable es que el conocimiento de las asincronías y su solución son la clave del éxito.

\section{BIBLIOGRAFÍA}

1. de Haro C, Ochagavia A, López-Aguilar J, Fernandez-Gonzalo $S$, Navarra-Ventura G, Magrans R, et al. Patient-ventilator asynchronies during mechanical ventilation: current knowledge and research priorities. Intensive Care Med Exp. 2019;7(Suppl $1): 43$.

2. Thille AW, Rodriguez P, Cabello B, Lellouche F, Brochard L. Patient-ventilator asynchrony during assisted mechanical ventilation. Intensive Care Med. 2006;32(10):1515-1522.

3. Gordo-Vidal F, Lobo-Valbuena B. SOS asynchronies: do we need help?* Critical Care Medicine. 2018;46(9):1549-1550.

4. Blanch L, Villagra A, Sales B, Montanya J, Lucangelo U, Luján M, et al. Asynchronies during mechanical ventilation are associated with mortality. Intensive Care Med. 2015;41(4):633-641.

5. Murias G, Lucangelo U, Blanch L. Patient-ventilator asynchrony. Curr Opin Crit Care. 2016;22(1):53-59.

6. Sousa MLA, Magrans R, Hayashi FK, Blanch L, Kacmarek RM, Ferreira JC. EPISYNC study: predictors of patient- 
ventilator asynchrony in a prospective cohort of patients under invasive mechanical ventilation - study protocol. BMJ Open. 2019;9(5):e028601.

7. Bruni A, Garofalo E, Pelaia C, Messina A, Cammarota G, Murabito $\mathrm{P}$, et al. Patient-ventilator asynchrony in adult critically ill patients. Minerva Anestesiol. 2019;85(6):676-688.

8. Dres M, Rittayamai N, Brochard L. Monitoring patient-ventilator asynchrony. Curr Opin Crit Care. 2016;22(3):246-253.

9. Holanda MA, Vasconcelos RDS, Ferreira JC, Pinheiro BV. Patientventilator asynchrony. J Bras Pneumol. 2018;44(4):321-333.

10. Subirà C, de Haro C, Magrans R, Fernández R, Blanch L. Minimizing asynchronies in mechanical ventilation: current and future trends. Respir Care. 2018;63(4):464-478.

11. Messina A, Colombo D, Cammarota G, De Lucia M, Cecconi $\mathrm{M}$, Antonelli $\mathrm{M}$, et al. Patient-ventilator asynchrony affects pulse pressure variation prediction of fluid responsiveness. J Crit Care. 2015;30(5):1067-1071.

12. Garofalo E, Bruni A, Pelaia C, Liparota L, Lombardo N, Longhini $F$, et al. Recognizing, quantifying and managing patient-ventilator asynchrony in invasive and noninvasive ventilation. Expert Rev Respir Med. 2018;12(7):557-567.

13. Pham T, Telias I, Piraino T, Yoshida T, Brochard LJ. Asynchrony consequences and management. Crit Care Clin. 2018;34(3):325-341.

14. Vaporidi K, Babalis D, Chytas A, Lilitsis E, Kondili E, Amargianitakis $\mathrm{V}$, et al. Clusters of ineffective efforts during mechanical ventilation: impact on outcome. Intensive Care Med. 2017;43(2):184-191.

15. de Haro C, López-Aguilar J, Magrans R, Montanya J, FernándezGonzalo S, Turon M, et al. Double cycling during mechanical ventilation: frequency, mechanisms, and physiologic implications. Crit Care Med. 2018;46(9):1385-1392.

16. Ruiz-Ferrón F, Serrano-Simón JM. Identificación del trigger inverso en UCl. Med Instensivea. 2018;42(6):391-393.

17. Rolland-Debord C, Bureau C, Poitou T, Belin L, Clavel M, Perbet $S$, et al. Prevalence and prognosis impact of patient-ventilator asynchrony in early phase of weaning according to two detection methods. Anesthesiology. 2017;127(6):989-997.

18. Longhini F, Colombo D, Pisani L, Idone F, Chun P, Doorduin J, et al. Efficacy of ventilator waveform observation for detection of patient-ventilator asynchrony during NIV: a multicentre study. ERJ Open Res. 2017;3(4). pii: 00075-2017.

19. Beloncle F, Piquilloud L, Rittayamai N, Sinderby C, Rozé $\mathrm{H}$, Brochard L. A diaphragmatic electrical activity-based optimization strategy during pressure support ventilation improves synchronization but does not impact work of breathing. Crit Care. 2017;21(1):21.

20. Goligher EC. Synchrony and the art of mechanical ventilation. Anesthesiology. 2017;127(6):915-917.

\section{Correspondencia:}

Alexéi Humberto Morales Morales

Av. Instituto Politécnico Nacional Núm. 5160,

Col. Magdalena de las Salinas,

Del. Gustavo A. Madero, 07760,

Ciudad de México, México.

Tel: 9612337673

E-mail: alexeimormorales@gmail.com

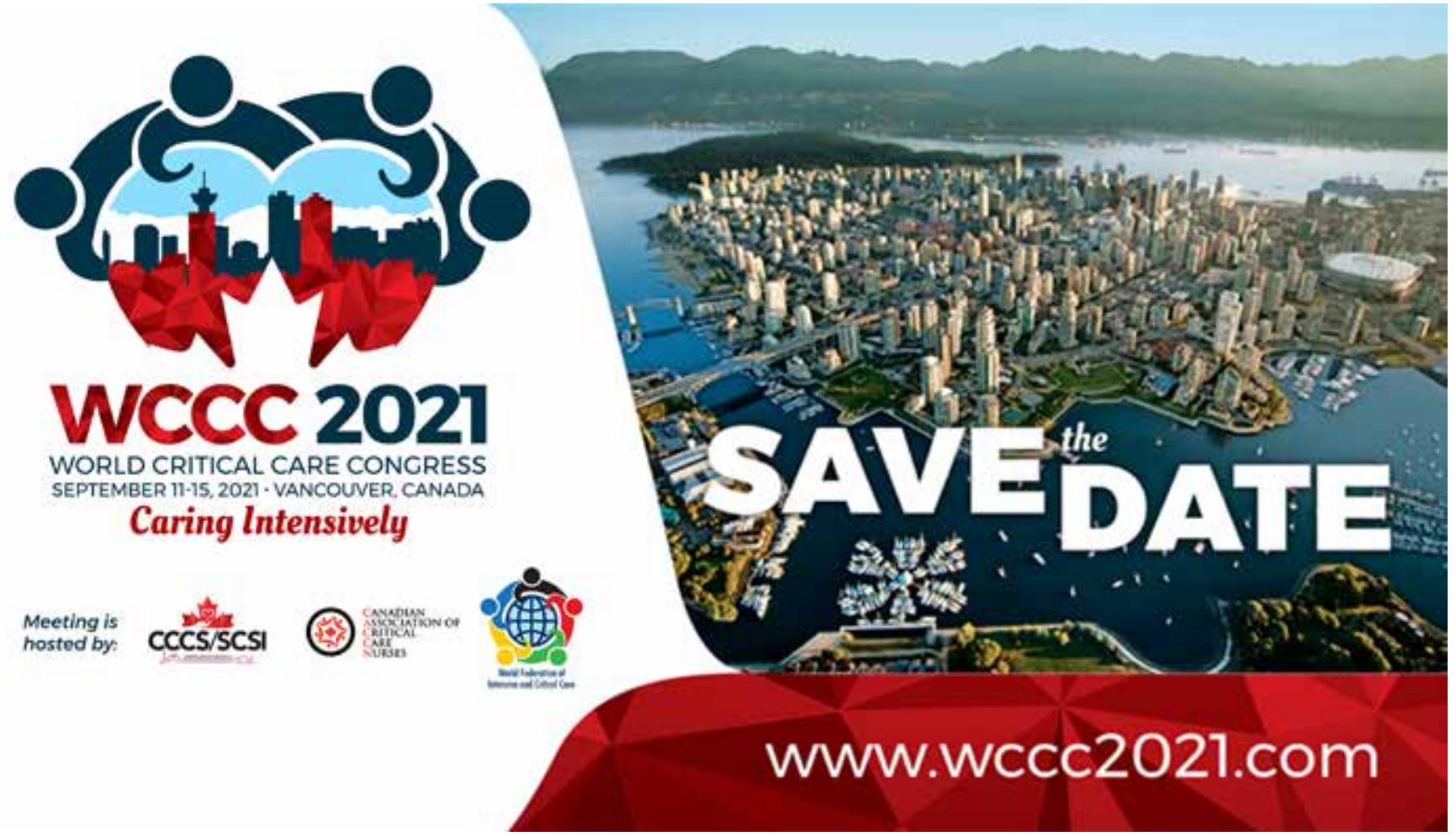

